\title{
2 Impact of visual features on capture of Aedes aegypti with host decoy traps (HDT)
}

4 Joseph Y. Tang ${ }^{1}$, Jackline Kosgei², Eric Ochomo ${ }^{2}$, Bryson A. Ndenga ${ }^{2}$, Roya Ghiaseddin ${ }^{3}$, Neil F.

5 Lobo $^{1}$, Frances M. Hawkes ${ }^{4}$, Joseph E. O’Tousa ${ }^{1}$

$7 \quad{ }^{1}$ Eck Institute for Global Health and Department of Biological Sciences, University of Notre

8 Dame, Notre Dame, IN, USA

$9 \quad{ }^{2}$ Kenya Medical Research Institute Centre for Global Health Research, Kisumu, Kenya

$10 \quad{ }^{3}$ Department of Applied and Computational Mathematics and Statistics, University of Notre

11 Dame, Notre Dame, IN, USA

$12{ }^{4}$ Natural Resources Institute, University of Greenwich at Medway, Chatham Maritime, Kent ME4

$13 \quad 4 \mathrm{~TB}, \mathrm{UK}$

15 Corresponding Author: Joseph E. O’Tousa, email: jotousa@nd.edu, phone 574-631-6093. Full address:

16 Department of Biological Sciences, University of Notre Dame, Notre Dame, IN, USA 46556

18 Running head: Visual cues in mosquito trap performance

Keywords: Mosquito visual behavior, Vector surveillance, Host Decoy Trap, Mosquito vision

\section{Abstract}

The host decoy trap (HDT) is a surveillance trap that presents a combination of heat, visual,

24 and odor stimuli to attract bloodmeal-seeking mosquitoes. Here we employed a semi-field study to 
25 demonstrate the role of the visual attributes present on the HDT on the effectiveness of Aedes aegypti

26 capture. Our results show that the HDT is an effective means of capturing Ae. aegypti mosquitoes in

27 semi-field conditions, with a per trial capture rate of up to $69 \%$ across four visually distinct HDTs. The

28 solid black colored HDT captured more mosquitoes than HDTs with black-white stripes, black-white

29 checkerboard patches, or solid white color by a factor of $1.9,1.7$, and 1.5 respectively. In all cases,

30 mosquito capture was not evenly distributed on the HDT surface, with captures on the HDT's outer

31 downwind half, away from the odor delivery, exceeding captures on the inner upwind half. We

32 conclude that the solid black surface of the original HDT design is more effective than the other

33 surfaces (white or black/white patterns) for the capture of Ae. aegypti. Our results demonstrate that

34 mosquito attraction to the thermal and odorant cues of the HDT is modulated by visual information.

35

36

\section{Introduction}

The oppressive human diseases of yellow fever, dengue fever, chikungunya, and Zika are all vectored by the Aedes aegypti mosquito, creating a heavy disease burden in Ae. aegypti-endemic regions (Bhatt et al., 2013; Musso et al., 2015). Control of Ae. aegypti and other vector species include insecticide-treated nets (ITN), indoor-residual spraying (IRS), and larvicide techniques. Interventions such as these knock down local mosquito populations, thereby curbing the transmission of a number of mosquito-borne diseases (Hawley et al., 2003; Mabaso et al., 2004; Weeratunga et al., 2017; CheMendoza et al., 2018).

Aedes aegypti's geographical distribution necessarily informs the application of vector control. Aedes species traditionally were considered to propagate in tropical and subtropical climates but recently have expanded into temperate regions (Kraemer et al., 2015). Global climate change and 
exploding rates of transcontinental human travel and interconnectedness could benefit Ae. aegypti and

49 its propagation worldwide, necessitating diligent vector surveillance and refinement of distribution maps (Brown PT; Glaesser et al., 2017).

Effective surveillance traps apply principles of Ae. aegypti's navigation, oviposition, and hostseeking mechanisms. With respect to female host-seeking, laboratory studies have described the effects

53 of a range of sensory stimuli on host-seeking behaviors. The major contributor to long-range host

54 detection is the olfactory system, with $\mathrm{CO}_{2}$ plumes originating from hosts dictating the flight direction of Ae. aegypti females from a distance (Carde, 2015). Once the mosquitoes have closed the distance to the source of stimuli, however, visual and thermal cues begin to play a significant role, with dark, warm, and visually contrasting objects being most attractive to the Ae. aegypti females (Wood \& Wright, 1968; Muir et al., 1992; van Breugel et al., 2015). Moreover, studies have shown that these cues act in concert. For instance, $\mathrm{CO}_{2}$ detection is a prerequisite for female mosquitoes' responses to host-derived odors such as lactic acid (McMeniman et al., 2014), whereas the detection of dark visual cues is a prerequisite for thermotaxis (Liu \& Vosshall, 2019). Although male Ae. aegypti do not exhibit host-seeking drives, their ability to navigate towards a target has been shown to be influenced by a similar set of stimuli, including a positive correlation with the visual complexity of the target's environment (Staunton et al., 2020).

Surveillance traps make use of multiple sensory cues relevant to the behavior of target mosquitoes that is induced by particular physiological statuses. One such trap, the host decoy trap (HDT), primarily targets female mosquitoes seeking human targets for bloodmeals. The HDT, in its original design, is a black cylindrical structure presenting a warm surface and the odors of a live host to mosquitoes. The surface of the HDT is adhesive, allowing mosquitoes to be captured if they land on the HDT. The effectiveness of the HDT has been established for the capture of the malaria mosquito 
71 Anopheles gambiae s.l., as well as Mansonia and Culex species (Abong'o et al., 2018). Iyaloo, et al.

72 (Iyaloo et al., 2017) demonstrated Aedes albopictus are captured by a different multisensory

73 cylindrical trap, the BG-Sentinel. In the latter study, traps composed of black cylinders and black lids

74 were shown to capture more mosquitoes than traps containing white cylinders/lids and traps presenting

75 contrasting black and white colors on the cylinder and lids.

76 In this study, we evaluated both the effectiveness of the HDT as a means of capturing Ae.

77 aegypti and the effect of different visual stimuli on the HDT surface in attracting Ae. aegypti. To

78 determine this, we compared the capture rates of four HDTs with varying visual appearances. These

79 alterations included a solid black surface, a black-white striped surface, a black-white checkerboard

80 surface, and a solid white surface. In doing so, this study demonstrates the importance of visual cues in

81 the host-seeking behavior of Ae. aegypti.

82

83

Materials and Methods

84 Study Site. This study was conducted during May and June 2019, using semi-field screen house arenas

85 at the Kisian Campus, Centre for Global Health Research (CGHR) of the Kenya Medical Research

86 Institute (KEMRI) in Kisian, Kisumu County, Kenya.

87 Setup of Host Decoy Traps. All HDTs used in this study were manufactured by BioGents AG and

88 used primarily as described in Abong'o et al. (2018) with the following modifications. In addition to

89 the original solid black HDT, we employed HDTs with a solid white surface (HDT W), a striped

90 pattern consisting of evenly alternating black and white stripes each of $6 \mathrm{~cm}$ width (HDT S), and a

91 checkerboard pattern consisting of $6 \mathrm{~cm} \times 6 \mathrm{~cm}$ alternating black and white square patches (HDT P).

92 To create HDT S and P, the black areas were printed onto the white vinyl fabric, and the fabric

93 wrapped around, and also placed on the upper surface, of each of the drums (Figure 1A). HDT W was 
94 created using the same white vinyl fabric. The sides of all four drums were then wrapped with

95 transparent adhesive plastic sheets (FICS Film, Barrettine Environmental Health, Bristol, UK) so that

96 identical tactile surfaces able to capture mosquitoes were present on all HDTs.

97 Semi-field arena. The arena used in this study was constructed using untreated Optinet netting

98 material permeable to air and draped over a $20 \mathrm{~m}$ long $\mathrm{x} 8 \mathrm{~m}$ wide $\mathrm{x} 3 \mathrm{~m}$ high galvanized steel frame.

99 Figure 1B depicts the arrangement of PVC pipes and connectors directing airflow to the four HDTs in

100 the arena. To ensure that the sensory cues of each HDT were as spatially distinct as possible, the traps

101 were symmetrically positioned such that the distance between each pair of HDTs was at least $4 \mathrm{~m}$, and

102 that the distance between the HDTs and the arena walls was at least $2 \mathrm{~m}$. Mosquitoes were released

103 from the center of the arena, equidistant from each of the HDTs. Due to the terrain over which the

104 semi-field arena was constructed, the four corners of the arena possessed different vegetation cover and

105 soil types. Moreover, the direction of the sunset cast varying levels of light intensity upon each trap

106 position. To minimize the impact of these and other variables on observed capture rates, we employed

107 a Latin square design in which all four HDTs were systematically rotated to different positions for each

108 trial such that each HDT was placed at each position the same number of times.

109 Natural host odors were transferred to the HDTs from a ventilated canvas tent (Pop Up Tent,

110 Sports God) $2 \mathrm{~m}$ outside the arena (Figure 1C). The same human volunteer sat in the tent for the

111 duration of each of the 16 trials conducted. A high-speed $12 \mathrm{~V}$, DC fan (Delta) powered by a $12 \mathrm{~V}, \mathrm{DC}$

1127 ah rechargeable lead acid batteries (ExpertPower) was attached via duct tape to a PVC pipe $(10 \mathrm{~cm}$ in

113 diameter). This end of the pipe was placed inside the tent. The PVC pipe was directed into the arena

114 and sealed at its entry point with cement. Each of the four pipe exit points was sealed with untreated

115 mosquito netting. An HDT unit was positioned $10 \mathrm{~cm}$ away from each opening to allow odors and $\mathrm{CO}_{2}$

116 from the tent to ventilate over each HDT. The $12 \mathrm{~V}$ fan provided a wind speed of approximately 1.26 
$117 \mathrm{~m} / \mathrm{s}$ at each exit point, delivering approximately $600 \mathrm{l} / \mathrm{min}$ of human odors to each HDT. These

118 parameters were set to approximate those used by Abong'o et al. (Abong'o et al., 2018).

119 Mosquito Rearing. Aedes aegypti mosquitoes present at sites around Kisian, Kisumu County were

120 captured using ovitraps and used to establish a breeding colony within the rearing facility. Larvae were

121 fed on Super Brewers Yeast Tablets (Pharmadass Ltd., Healthaid House, Marlborough Hill, Harrow,

122 Middlesex, HA1 1UD, United Kingdom) ground into powder and deposited in water as required. The

123 resulting F1 male and female adults were fed a 10\% solution of sugar. These adults were aged for five

124 days to provide an opportunity for females to mate. The sugar solution was removed 6 hours prior to

125 the collection of females.

126 Trial Operation and Data Collection. KEMRI insectary staff maintained production of adult mosquito

127 rates sufficient to provide 200 female mosquitoes per trial, the same number used in previous semi-

128 field studies (Dugassa et al., 2014; Batista et al., 2017). One hundred females were aspirated into each

129 of two paper cups (a total of 200) before each experimental trial and transported to the arena. Each

130 HDT was heated with hot water as described by Abong'o et al. (2018). At the start of each trial, the

131 temperature of the HDT surface, the wind speeds at pipe exit points, the light intensity in the arena, and

132 the arena's temperature and humidity were measured and recorded using an infrared spot thermometer,

133 anemometer, photometer, and a temperature-humidity sensor respectively. The mosquitoes were then

134 released from the center of the screen house (Figure 1C). To align with the day-biting behavior of Ae.

135 aegypti, each trial began between 12:00 $\mathrm{h}$ and 13:00 h, and ended at 18:00 h, lasting a total of 5-6

136 hours. The same set of HDT-specific and environmental parameters were re-measured at the

137 conclusion of each trial. Mosquitoes not captured on the HDTs during the trials were collected from the 138 arena using a battery-powered aspirator (Prokopack Model 1419, The John W. Hock Company). 
At the end of each trial, the adhesive sheet on the surface of each HDT was wrapped with

140 plastic food wrap, sandwiching the captured mosquitoes between the adhesive sheet and the wrap. The

141 adhesive sheets were labeled using permanent marker to denote the HDT type, the position of the black

142 and white regions on the striped and patched HDTs, and the location of the pipe outlet.

143 Data Analysis. Mosquitoes captured on the HDT adhesive sheets and recaptured from the arena were

144 killed in a $-20^{\circ} \mathrm{C}$ freezer overnight. The number of captured mosquitoes on each HDT type were then

145 counted. For all adhesive sheets, the count of mosquitoes on each HDT's inner half (facing the pipe

146 outlet) and outer half (facing away from the pipe outlet) was determined. For HDTs S and P, the count

147 of mosquitoes captured on their black and white regions was also determined.

148 The data were analyzed using SPSS Statistics software, version 26.0.0. Confounding variables

149 were assessed via ANOVA (analysis of variance) and Kruskal-Wallis analyses. Linear regression

150 assessed the effect of ambient environmental variables, odor wind speeds, and HDT surface

151 temperatures on the catch of mosquitoes on HDTs. To address the primary research question (i.e.,

152 which HDT type had the highest capture rate), $\chi^{2}$ goodness of fit analysis determined if differences in

153 capture rates between the HDT types were statistically significant. Then, pairwise post hoc

154 comparisons between the traps were conducted among pairs of HDT types to determine the source of

155 the capture rate incongruence, and thus statistical differences between HDT types. Similar pairwise

156 comparisons were made to determine whether there were landing preferences on the black and white

157 regions of the striped and patched HDTs ( $\mathrm{S}$ and $\mathrm{P}$ ). An independent samples comparison was used to

158 assess mosquito preference for landing on the outer half of the HDT surface. 
162 summarizes the capture data from the 16 replicates of this study. Of the total of 3200 released

163 mosquitoes, 1096 (34\%) were captured by the HDTs. $\chi^{2}$ goodness of fit analysis of the capture counts

164 of the four HDT types revealed that there is a significant overall difference between the HDT types'

165 mean capture rates $\left(\chi^{2}=77.82, p=9.014 \times 10^{-17}\right)$, and 11 out of $16 \chi^{2}$ GOF tests on individual

166 trials revealed significant differences in the HDTs' capture rates (Table 1).

HDT B captured the most mosquitoes, with a mean of 24.81 per trial (95\% CI: $18.54-31.09$ )

168 compared to the 12.81 captured by HDT S (95\% CI: 9.14-16.49), the 14.81 by HDT P (95\% CI: 10.49 -

169 19.13), and the 16.06 by HDT W (95\% CI: 12.44-19.69) (Figure 2A). Paired analyses of differences in

170 captures amongst HDT types in each trial revealed that HDT B's capture counts significantly exceeded

171 those of HDTs S, P, and W by a mean difference of 12 (95\% CI: 6.6-17.4), 10 (95\% CI: 5.3-14.7), and

1728.75 (95\% CI: 2.7-14.8) respectively (Figure 2B). These analyses revealed no significant differences

173 between the mean capture counts per trial between HDTs S, P, and W (Figure 2B).

174

Aedes aegypti showed no preference for landing on black areas of HDT S and HDT P. We

176 separately counted the captures on the black and white areas of HDTs S and P to determine if Aedes

177 mosquitoes showed a preference for landing on one of these surfaces. $\chi^{2}$ goodness of fit analysis of

178 HDT P's capture counts on the black patches (84 captured) and white patches (85 captured) indicated

179 no significant difference in captures $\left(\chi^{2}=0.0059, p=0.9387\right)$. The same analysis performed on

180 HDT S showed a modest trend towards increased capture within the black stripes (133 captured)

181 relative to the white stripes (107 captured) but this difference was not statistically significant $\left(\chi^{2}=\right.$

$1822.8167, p=0.0933)$.

183 
184 Ae. aegypti prefers to land on the outer half of the HDT. During post-trial analysis, the location of

185 each captured mosquito was categorized by whether they landed on the inner, facing the odor delivery,

186 or outer half of the HDT that faced away from the odor delivery site (Figure 3A). Trials 1 and 3 were

187 excluded from this analysis due to heavy rain that washed away the labels on the sticky sheets. In the

188 remaining 14 trials, the distribution of Ae. aegypti landings on the HDTs' surfaces indicated significant

189 skew towards the outer half landings compared to the inner half landings for each HDT type (Figure

190 3B). The inner halves of HDTs B, S, P, and W caught an average of 4.36, 3.36, 2.94, and 2.79

191 mosquitoes respectively, whereas the outer halves of B, S, P, and W caught 20.79, 12.50, 10.86, and

19214.29 respectively. In total, capture on the outer halves was significantly greater than capture on the

193 inner halves ((11.25 mosquitoes/trial, 95\% CI: $9.05-13.45, p<0.0001)$. A similar analysis

194 performed individually for each HDT type also showed a significant excess of captures on the outer 195 halves.

197 Capture of Ae. aegypti by HDTs was not significantly affected by environmental variables. To

198 determine if the position of the HDT within the screen house influenced capture rates, the data for all

199 HDT types were stratified by location (Table 2). When no distinction was made with respect to the

200 HDT type at each position, the differences in captures at each position was not significant $(F=$

$2012.185, p=0.099)$. For three of the four HDT types, differences in capture rate based on position in the

202 screen house showed no statistical significance. The fourth, HDT S, did show a significant difference

203 in capture between positions 2 and 3, the positions closest to the entrance of the arena. However, given

204 the lack of statistical significance for all other tests involving positions 2 and 3, this finding was not

205 expected to alter conclusions regarding the mosquito's attraction to HDT trap type. 
We found no evidence that ambient light intensity, temperature, and humidity conditions

207 influenced the total mosquito capture during the 16 trials. Linear regression analysis indicates no 208 significant correlation between a trial's total HDT capture and: (1) the mean light intensity ( $p=$

209 0.697), (2) the trial's mean ambient temperature ( $p=0.669)$, and (3) the mean relative humidity ( $p=$

210 0.521) respectively. Similarly, a lack of significant correlation was observed with regression analyses

211 of the values of each of these environmental variables at the beginning and end of each trial. Moreover,

212 for each HDT type, no correlation was observed between capture counts and the mean surface

213 temperatures ( $p=0.157)$ and wind speed $(p=0.853)$ at the pipe exit points.

\section{Discussion}

The goal of this study was to characterize the impact of altering visual characteristics on the

217 landing frequency of Ae. aegypti females. The HDT paradigm allows for a host mimic target to present

218 variations in the parameters of host-associated stimuli. The original HDT design outlined in Hawkes et

219 al. (Hawkes et al., 2018) used a solid black surface color. This choice is supported by laboratory

220 studies that point to Ae. aegypti's visual preferences. Muir et al. (1992) demonstrated Ae. aegypti's

221 preference for landing on dark targets. More recent studies uncovered details on the interactions

222 between different types of stimuli. Van Breugel et al. (2015) demonstrated that $\mathrm{CO}_{2}$ detection activates

223 a strong attraction for dark targets on a light background. Liu \& Vosshall (2019) also showed Ae.

224 aegypti's preference for landing on a single dark spot on light background in the presence of $\mathrm{CO}_{2}$.

225 These studies suggested that contrasting surface features might enhance the HDT's effectiveness. To

226 test this, we designed HDTs having different visual features but possessing identical nonvisual cues

227 (detectable heat signature, host odors, and $\mathrm{CO}_{2}$ ) known to attract Ae. aegypti (van Breugel et al., 2015;

228 Liu \& Vosshall, 2019). 
230 The original black HDT showed the highest capture rates. Our results indicated that the black surface 231 was more effective than either the white surface or the black/white contrasting surfaces of the S and P 232 HDTs. Importantly, these results establish that the Ae. aegypti attraction to the HDT is influenced by 233 the visual attributes of the trap. However, the results are contrary to our expectation in that the $\mathrm{S}$ and $\mathrm{P}$ 234 HDTs would improve the HDT's capture rate. These two HDTs were outperformed by both HDT B and

235 HDT W. One consideration is that the width of the stripes and patches were set at $6 \mathrm{~cm}$ on the $\mathrm{S}$ and $\mathrm{P}$ 236 HDTs. Ae. aegypti requires a minimum optical angle of between $4^{\circ}$ and $8^{\circ}$ to perceive distinct objects 237 (Bidlingmayer, 1994). At a distance of $1 \mathrm{~m}$, the angle of perception of a $6 \mathrm{~cm}$ wide stripe is $3.4^{\circ}$. For 238 this reason, the black/white regions of these traps are not likely to be visible to a mosquito until they 239 are less than one meter away from the trap. At greater distances, the solid black trap would present the 240 strongest contrast to the surrounding environment than the other HDTs. Thus, it is possible that HDT

241 performance could be improved by increasing the size of the contrasting black and white regions so 242 that Ae. aegypti could discern the contrasting surface of the HDT at greater distances.

244 Mosquito capture was not evenly distributed on the HDT. In this study we found a greater concentration of mosquitoes captured on the HDTs outer half, facing away from the odor delivery 246 pipe. This suggests that the three-dimensional surface of the HDT was not a uniform surface for 247 capture. Clearly there was a nonuniform distribution of odors across the surface of the HDTs since the 248 odors were vented from a single exit point directed toward the HDT. Because odors were deposited at $24910 \mathrm{~cm}$ away from the base of the HDT, the surface of the HDT facing the exit point received the odor 250 immediately as it was dispensed from the pipe. An important consideration is that the odor was vented 251 from the pipe at a speed of approximately $1.26 \pm 0.18 \mathrm{~m}^{-\mathrm{s}}$, which may have created sufficient 
252 directional air flow to induce upwind flight in Ae. aegypti. Geier et al. (1999) showed that A. aegypti

253 increase their upwind flight activity upon exposure to plumes of both $\mathrm{CO}_{2}$ and host odors both

254 independently and in combination. Because odor delivery through the pipe creates a wind current,

255 mosquitoes would have first encountered the HDT surface facing away from the pipe due to this

256 tendency to fly upwind while tracking odor plumes. Additionally, the wind velocity resulting from

257 airflow out of the pipe may have limited the ability of the mosquito to reach the surface facing the

258 pipe. Further investigation of the dynamics of airflow onto and around the HDT's cylindrical drum

259 may illuminate these mechanisms as well as alternative methods of odor delivery to improve overall

260 capture rates.

262 The HDT is a useful tool for both mosquito surveillance and behavioral studies. This study

263 investigated the role of visual stimuli on HDT capture of female Ae. aegypti likely engaged in host-

264 seeking behavior. The compact materials of the HDT allows it to be transported to the vicinities of

265 remote human communities with difficult terrains and climates where surveillance of mosquito

266 prevalence is most lacking, and little supervision is required once set up is complete. Data provided by

267 the HDT can effectively serve as important indicators of regional species prevalence and host

268 preferences and will contribute to further refinements of probability models of Ae. aegypti and other

269 species' geographical distributions (Kraemer et al., 2015). The importance of such models was

270 demonstrated in a study that aligned the known distributions of several Aedes species throughout

271 Thailand and the distributions of human incidences of dengue, chikungunya, and Zika infections

272 (Suwanmanee et al., 2018). Continuously updating information on vector prevalence is crucial to

273 combatting short-term disease outbreaks and to observing long-term trends in geographical shifts in

274 vector species distribution. 
279 HDTs have the intrinsic advantage over HLCs in that they present negligible risk of mosquito bites to 280 researchers. The properties of the HDTs can also be modified to present different stimuli that may arise 281 from hosts, manipulating experimental variables related to thermal, olfactory and visual properties of 282 hosts so that the effect of these properties on host-seeking behavior of field populations can be studied. 283 We have demonstrated the HDT's ability to safely and effectively capture Ae. aegypti females, extending the work of Abong'o et al. (2018) showing effective capture of Anopheles and Culex species 285 in field settings.

The presence of attractive visual cues on traps can contribute to mosquito control. Many current

288 vector control methods such as insecticide-treated bed nets (ITNs) and indoor residual spraying (IRS), 289 though effective at hampering human contact with mosquitoes, do not eliminate mosquito populations 290 (van den Berg, 2011). The sustained use of insecticides consistently leads to mosquito resistance 291 (Barrera et al., 2013; Moyes et al., 2017; Dusfour et al., 2019). Mosquito surveillance traps could be 292 adapted as alternative vector control tool. The BG-Sentinel, for example, is a multisensory surveillance 293 trap that was shown to be effective at controlling populations of Ae. mediovittatus, a mosquito vector 294 native to the Caribbean (Barrera et al., 2013). Moreover, with regards to its visual design, Iyaloo et al. 295 (Iyaloo et al., 2017) demonstrated Ae. albopictus' preference for landing on a solid black BGS over 296 other black-white surface patterns. Similarly, optimizing the HDT's visual design could be an avenue 297 of introducing it as a vector control tool. This study's findings constitute a step towards not only 
optimizing the visual design of the HDT, but also elucidating general patterns of vision-based behavior

299 that can inform the design of novel vector control methods. Ultimately, any vector control methods that

300 take advantage of sensory cues instead of or in addition to the traditional chemical cues would

301 diversify the set of stimuli that place selective pressure on mosquito evolution and mitigate the

302 development of resistance to vector control (Amelia-Yap et al., 2018).

303

304

Data Availability Statement

305 The raw data that were reported as averages in this study are available from the corresponding author 306 upon reasonable request.

\section{Acknowledgments}

309 This study was supported by the Eck Institute for Global Health at the University of Notre Dame, 310 National Institutes of Health award 1R21AI125765 (to JEO) and the Medical Research Council grant 311 MR/P025404/1 (to FMH). We recognize the contributions of James Oppalla and Austin Dawa for 312 assistance with the operation of trials, Peter Aswani for mosquito rearing services, and Lara Lontoc for assistance with illustrations. We thank the KEMRI Director General for granting permission for this manuscript's publication. The authors confirm ownership of the presented data and that all contributors have been appropriately identified.

\section{References}

Abong'o, B., Yu, X., Donnelly, M. J., Geier, M., Gibson, G., Gimnig, J., et al. (2018) Host Decoy Trap (HDT) with cattle odour is highly effective for collection of exophagic malaria vectors. Parasit Vectors, 11, 533.

Amelia-Yap, Z. H., Chen, C. D., Sofian-Azirun, M., Lau, K. W., Suana, I. W., Harmonis, et al. (2018) Efficacy of Mosquito Coils: Cross-resistance to Pyrethroids in Aedes aegypti (Diptera: Culicidae) From Indonesia. J Econ Entomol, 111, 2854-2860.

Barrera, R., Mackay, A. J. \& Amador, M. (2013) An improved trap to capture adult containerinhabiting mosquitoes. J Am Mosq Control Assoc, 29, 358-368.

Batista, E. P. A., Ngowo, H. S., Opiyo, M., Shubis, G. K., Meza, F. C., Okumu, F. O., et al. (2017) Semi-field assessment of the BG-Malaria trap for monitoring the African malaria vector, Anopheles arabiensis. PLoS One, 12, e0186696.

Bhatt, S., Gething, P. W., Brady, O. J., Messina, J. P., Farlow, A. W., Moyes, C. L., et al. (2013) The global distribution and burden of dengue. Nature, 496, 504-507. 
332 Bidlingmayer, W. L. (1994) How mosquitoes see traps: role of visual responses. J Am Mosq Control

333 Assoc, 10, 272-279.

334 Brown PT, C. K. (2017) Greater future global warming inferred from Earth's recent energy budget.

335 Nature, 552, 45-50.

336 Carde, R. T. (2015) Multi-Cue Integration: How Female Mosquitoes Locate a Human Host. Curr Biol,

337 25, R793-795.

338 Che-Mendoza, A., Medina-Barreiro, A., Koyoc-Cardena, E., Uc-Puc, V., Contreras-Perera, Y.,

339 Herrera-Bojorquez, J., et al. (2018) House screening with insecticide-treated netting provides sustained

340 reductions in domestic populations of Aedes aegypti in Merida, Mexico. PLoS Negl Trop Dis, 12,

341 e0006283.

342 Dugassa, S., Lindh, J. M., Torr, S. J., Lindsay, S. W. \& Fillinger, U. (2014) Evaluation of the influence

343

344

345

346

347

348 of electric nets on the behaviour of oviposition site seeking Anopheles gambiae s.s. Parasit Vectors, 7, 272.

Dusfour, I., Vontas, J., David, J. P., Weetman, D., Fonseca, D. M., Corbel, V., et al. (2019) Management of insecticide resistance in the major Aedes vectors of arboviruses: Advances and challenges. PLoS Negl Trop Dis, 13, e0007615.

Geier, M., Bosch, O.J., Boeckh, J. (1999) Influence of odour plume structure on upwind flight of mosquitoes towards hosts. J Exp Biol 202, 1639-1648.

Glaesser, D., Kester, J., Paulose, H., Alizadeh, A. \& Valentin, B. (2017) Global travel patterns: an overview. J Travel Med, 24, 1-5.

Hawkes, F. M., Sawadogo, S., Dabire, S., Torr, S. \& Gibson, G. (2018) Constructing a Host Decoy Trap for malaria vector sampling. dx.doi.org/10.17504/protocols.io.n95dh86.

Hawley, W. A., Phillips-Howard, P. A., ter Kuile, F. O., Terlouw, D. J., Vulule, J. M., Ombok, M., et al. (2003) Community-wide effects of permethrin-treated bed nets on child mortality and malaria morbidity in western Kenya. Am J Trop Med Hyg, 68, 121-127.

Iyaloo, D., Facknath, S. \& Bheecarry, A. (2017) Field evaluation of BG Sentinel ${ }^{\mathrm{TM}}$ traps of four different black-and-white color combinations in Mauritius for enhanced Ae. albopictus mosquito collection. Int Journal Mosquito Res, 4, 43-49.

Kraemer, M. U., Sinka, M. E., Duda, K. A., Mylne, A. Q., Shearer, F. M., Barker, C. M., et al. (2015) The global distribution of the arbovirus vectors Aedes aegypti and Ae. albopictus. Elife, 4, e08347. Liu, M. Z. \& Vosshall, L. B. (2019) General Visual and Contingent Thermal Cues Interact to Elicit Attraction in Female Aedes aegypti Mosquitoes. Curr Biol, 29, 2250-2257 e2254.

Mabaso, M. L., Sharp, B. \& Lengeler, C. (2004) Historical review of malarial control in southern African with emphasis on the use of indoor residual house-spraying. Trop Med Int Health, 9, 846-856. McMeniman, C. J., Corfas, R. A., Matthews, B. J., Ritchie, S. A. \& Vosshall, L. B. (2014) Multimodal integration of carbon dioxide and other sensory cues drives mosquito attraction to humans. Cell, 156, 1060-1071.

Moyes, C. L., Vontas, J., Martins, A. J., Ng, L. C., Koou, S. Y., Dusfour, I., et al. (2017)

Contemporary status of insecticide resistance in the major Aedes vectors of arboviruses infecting humans. PLoS Negl Trop Dis, 11, e0005625.

Muir, L. E., Kay, B. H. \& Thorne, M. J. (1992) Aedes aegypti (Diptera: Culicidae) vision: response to stimuli from the optical environment. J Med Entomol, 29, 445-450.

Musso, D., Cao-Lormeau, V. M. \& Gubler, D. J. (2015) Zika virus: following the path of dengue and chikungunya? Lancet, 386, 243-244. 
376 Staunton, K. M., Crawford, J. E., Cornel, D., Yeeles, P., Desnoyer, M., Livni, J., et al. (2020)

377 Environmental influences on Aedes aegypti catches in Biogents Sentinel traps during a Californian

378 "rear and release" program: Implications for designing surveillance programs. PLoS Negl Trop Dis, 14, 379 e0008367.

380 Suwanmanee, S., Surasombatpattana, P., Soonthornworasiri, N., Hamel, R., Maneekan, P., Misse, D., 381 et al. (2018) Monitoring arbovirus in Thailand: Surveillance of dengue, chikungunya and zika virus, 382 with a focus on coinfections. Acta Trop, 188, 244-250.

383 van Breugel, F., Riffell, J., Fairhall, A. \& Dickinson, M. H. (2015) Mosquitoes Use Vision to 384 Associate Odor Plumes with Thermal Targets. Curr Biol, 25, 2123-2129.

385 van den Berg, H. (2011) Global status of DDT and its alternatives for use in vector control to prevent 386 disease. Cien Saude Colet, 16, 575-590.

387 Weeratunga, P., Rodrigo, C., Fernando, S. D. \& Rajapakse, S. (2017) Control methods for Aedes 388 albopictus and Aedes aegypti. The Cochrane Database of Systematic Reviews, 8, CD012759.

389 Wood, P. \& Wright, R. (1968) Some responses of flying Aedes aegypti to visual stimuli. The Canadian 390 Entomologist, 100, 504-513.

391

392 
Table 1.

\begin{tabular}{|c|c|c|c|c|c|c|c|}
\hline HDT type & $\begin{array}{c}\text { Black } \\
\text { (B) }\end{array}$ & $\begin{array}{c}\text { Striped } \\
\text { (S) }\end{array}$ & $\begin{array}{c}\text { Patched } \\
(\mathbf{P})\end{array}$ & $\begin{array}{c}\text { White } \\
(\mathbf{W})\end{array}$ & $\begin{array}{c}\text { Total } \\
\text { captured on } \\
\text { HDTs }\end{array}$ & $\chi^{2}$ & p-value \\
\hline Trial 1 & 14 & 2 & 2 & 4 & 22 & 18.00 & $<\mathbf{0 . 0 0 1}$ \\
\hline Trial 2 & 40 & 17 & 16 & 17 & 90 & 18.18 & $<\mathbf{0 . 0 0 1}$ \\
\hline Trial 3 & 31 & 10 & 12 & 14 & 67 & 16.64 & $\mathbf{0 . 0 0 1}$ \\
\hline Trial 4 & 16 & 10 & 15 & 10 & 51 & 2.41 & 0.491 \\
\hline Trial 5 & 9 & 6 & 7 & 16 & 38 & 6.42 & 0.093 \\
\hline Trial 6 & 11 & 18 & 17 & 22 & 68 & 2.11 & 0.302 \\
\hline Trial 7 & 37 & 10 & 22 & 16 & 87 & 11.67 & $<\mathbf{0 . 0 0 1}$ \\
\hline Trial 8 & 36 & 30 & 12 & 17 & 95 & 6.32 & $\mathbf{0 . 0 0 1}$ \\
\hline Trial 9 & 18 & 14 & 9 & 17 & 57 & 3.38 & 0.337 \\
\hline Trial 10 & 20 & 11 & 15 & 19 & 65 & 3.12 & 0.373 \\
\hline Trial 11 & 24 & 4 & 15 & 10 & 52 & 16.21 & $\mathbf{0 . 0 0 1}$ \\
\hline Trial 12 & 32 & 18 & 16 & 14 & 80 & 10.00 & $\mathbf{0 . 0 1 9}$ \\
\hline Trial 13 & 11 & 10 & 5 & 23 & 49 & 14.27 & $\mathbf{0 . 0 0 3}$ \\
\hline Trial 14 & 49 & 19 & 36 & 34 & 138 & 13.13 & $\mathbf{0 . 0 0 4}$ \\
\hline Trial 15 & 23 & 9 & 25 & 15 & 72 & 9.11 & $\mathbf{0 . 0 2 8}$ \\
\hline Trial 16 & 26 & 17 & 13 & 9 & 65 & 9.77 & $\mathbf{0 . 0 2 1}$ \\
\hline Mean & 24.81 & 12.81 & 14.81 & 16.06 & $\mathbf{6 8 . 5}$ & 78.64 & $<\mathbf{0 . 0 0 1}$ \\
\hline Total & $\mathbf{3 9 7}$ & $\mathbf{2 0 5}$ & $\mathbf{2 3 7}$ & $\mathbf{2 5 7}$ & $\mathbf{1 0 9 6}$ & & \\
\hline & & & & & & & \\
\hline
\end{tabular}

397 Table 1. Compilation and $\chi^{2}$ GOF statistical analysis* of mosquito capture data in the 16 trials of the 398 study.

$399 * 11$ (bolded) out of 16 demonstrate significant difference in capture counts between the four HDTs.

400 All tests were performed with three degrees of freedom, with a null hypothesis of expected counts 401 being equal for all four HDT types.

402 
Table 2.

\begin{tabular}{|c|c|c|c|c|c|}
\hline Trap Position & Black (B) & Striped (S) & Patched (P) & White (W) & Total \\
\hline 1 & 52 & 65 & 56 & 55 & 228 \\
\hline 2 & 120 & 33 & 29 & 50 & 232 \\
\hline 3 & 115 & 75 & 84 & 60 & 334 \\
\hline 4 & 110 & 32 & 74 & 92 & 308 \\
\hline Total & 397 & 205 & 237 & 257 & 1096 \\
\hline $\begin{array}{c}\text { Statistical } \\
\text { Significance }\end{array}$ & $\begin{array}{c}\text { No } \\
(\mathrm{p}=0.112)\end{array}$ & $\begin{array}{c}\text { Yes } \\
(\mathrm{p}=0.031)\end{array}$ & $\begin{array}{c}\text { No } \\
(\mathrm{p}=0.149)\end{array}$ & $\begin{array}{c}\text { No } \\
(\mathrm{p}=0.107)\end{array}$ & \\
\hline
\end{tabular}

404

405

406

407

408

409

410

411

412 in capture due to trap position. Post-hoc tests determined that this difference was due to the HDT S

413 data collected from positions 2 and 3.

Table 2. Mosquito capture rates analyzed* with respect to position of the HDTs in the screen house.

*Two different statistical tests were used to determine if position was responsible for differences in capture rates. For HDT S and HDT W, because variances were equal at the four positions, ANOVA analysis was used, while for HDT B and HDT P, where the variances were unequal, Kruskal-Wallis was employed. The statistical analysis showed that only the HDT S data showed significant differences 

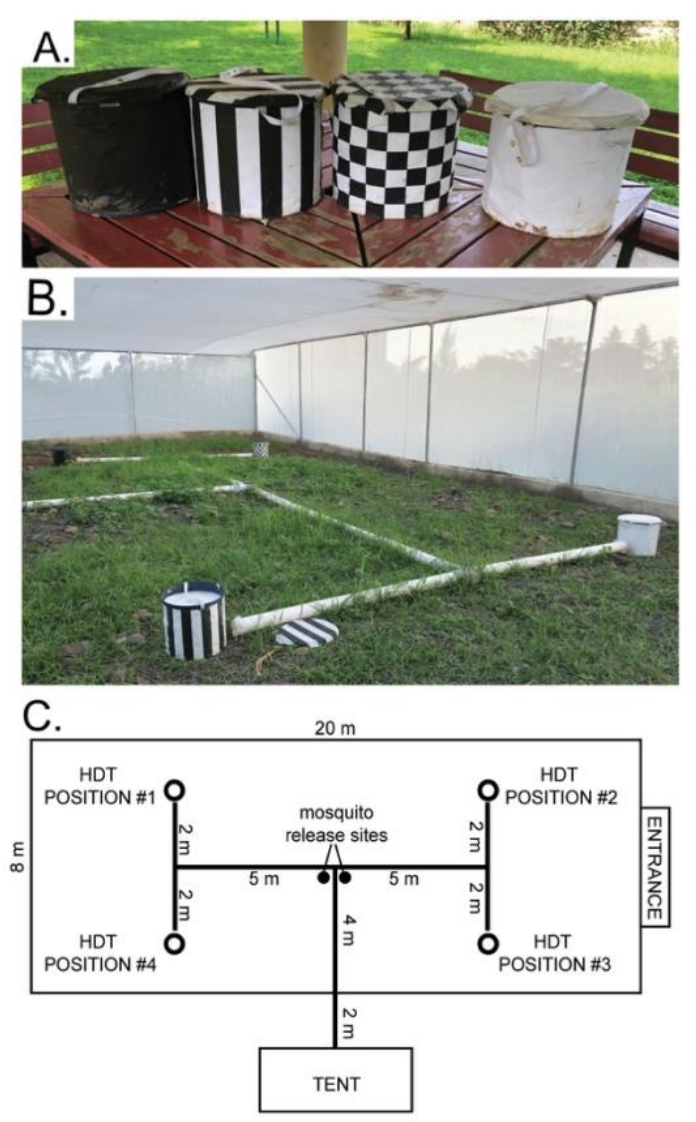

Figure 1.

418 Figure 1. Visual features on the HDTs and layout of the experimental facility.

419 (A) Test traps showing the visual feature designs for (left to right) the black, striped, patched and white 420 HDTs.

421 (B) Example arena set up of the four HDTs and the pipework delivering the human odors from the tent 422 to each of the HDTs.

423 (C) Diagram showing the dimensions of the screen house and other elements of the experimental set 424 up. A North arrow indicates the approximate cardinal orientation of the arena. The pipework delivers 425 odors from a human subject residing in the tent to symmetrically placed HDTs within the screen house. 


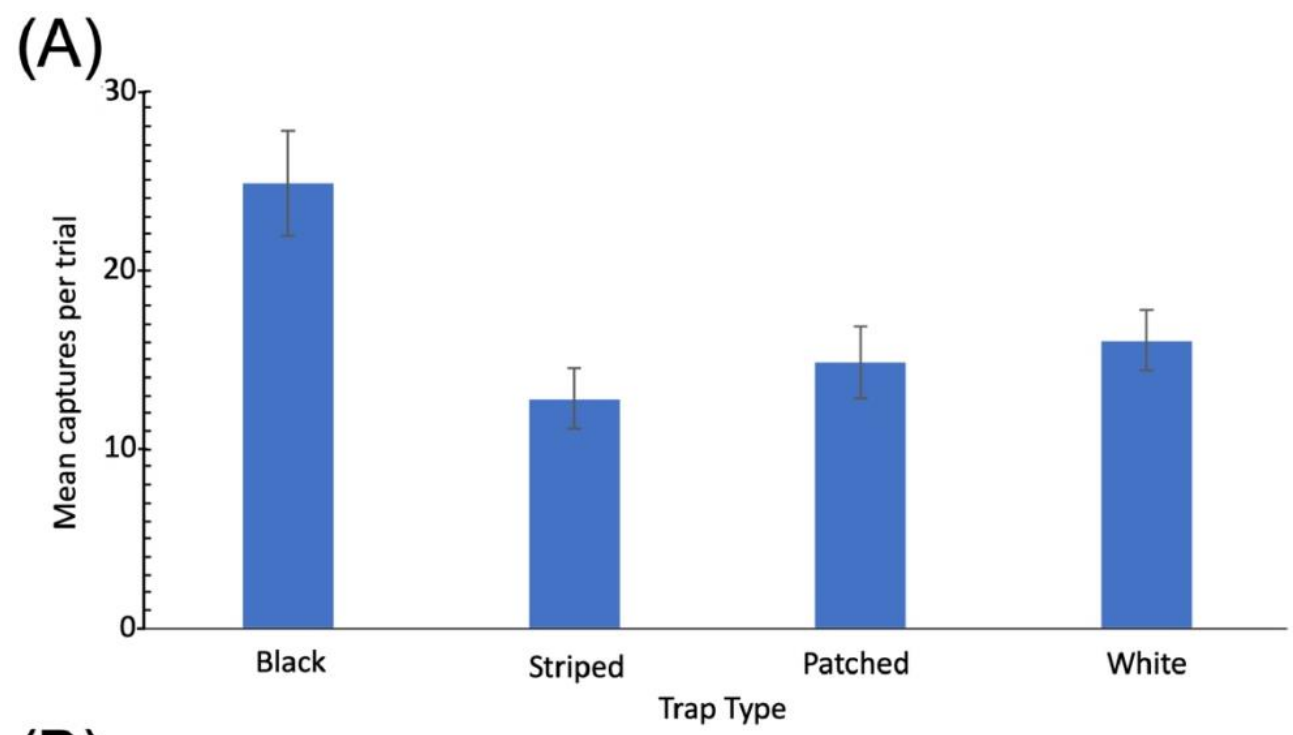

(B)

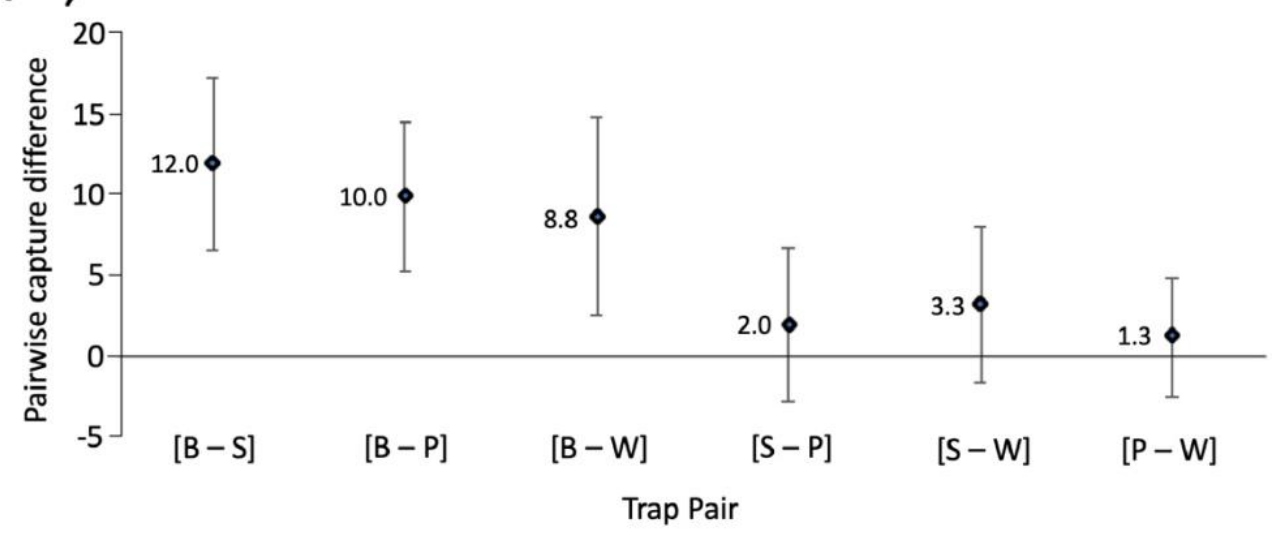

426 Figure 2.

427 Figure 2. Comparisons between mean capture rates of HDTs B, S, P, and W.

428 (A) Mean capture rates of each HDT type. Error bars indicate standard deviation.

429 (B) Pairwise differences in mean capture by trial between each pair of HDTs depicting the 95\%

430 confidence bands. Bands that do not include 0 indicate with $95 \%$ confidence that there is a non-zero

431 difference in captures between the pair of HDT types, i.e. a statistically significant difference. By this

432 metric, HDT B captured significantly more mosquitoes than the other three DHT types, while the

433 differences between HDTs S, P, and W are not significant. 


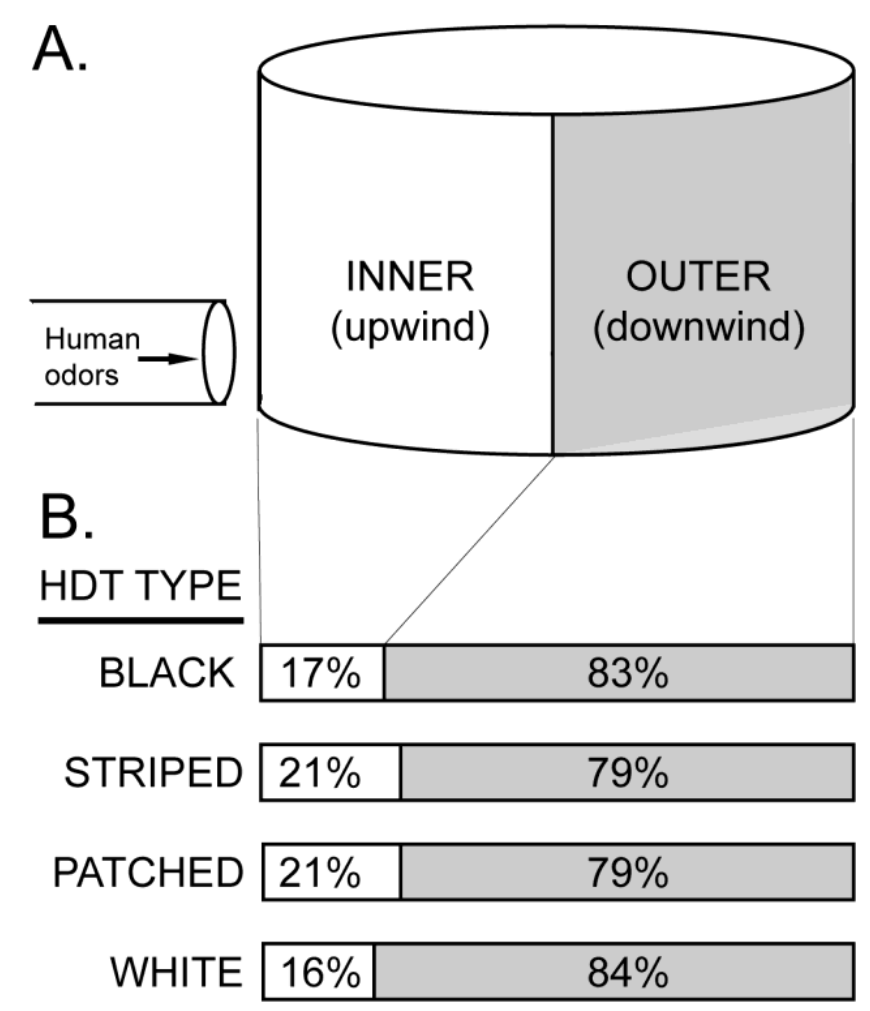

434 Figure 3.

435 Figure 3. Comparisons of mosquito capture on outer and inner halves of the HDTs.

436 (A) An illustration designating the inner and outer halves of the HDTs. The terms upwind and

437 downwind denote the direction of the wind currents generated by odor delivery through the pipe.

438 (B) The percent capture determined for the inner and outer surfaces of each HDT type. 

439 Abbreviations
440 KEMRI: Kenya Medical Research Institute
441 CGHR: Centre for Global Health Research
442 PVC: polyvinyl chloride
443 HDT: host decoy trap
$444 \quad$ HDT B: solid black HDT
445 HDT S: black/white striped HDT
446 HDT P: black/white patched HDT
447 HDT W: solid white HDT
448 HLC: human landing catch
$449 \quad \chi^{2}$ GOF: chi-square goodness of fit statistical test
450 RNA: ribonucleic acid
451 BGS: Biogents-Sentinel trap
452 ITN: insecticide-treated bed nets
453 IRS: indoor residual spraying
454 ANOVA: analysis of variance 